Rapport - Société canadienne d'histoire de l'Église catholique

\title{
Les Soeurs du Bon-Pasteur au Saguenay
}

\section{Imelda Côté}

Volume 32, 1965

URI : https://id.erudit.org/iderudit/1007327ar

DOI : https://doi.org/10.7202/1007327ar

Aller au sommaire du numéro

Éditeur(s)

Les Éditions Historia Ecclesiæ Catholicæ Canadensis Inc.

ISSN

0318-6148 (imprimé)

1927-7075 (numérique)

Découvrir la revue

Citer cet article

Côté, I. (1965). Les Soeurs du Bon-Pasteur au Saguenay. Rapport - Société canadienne d'histoire de l'Église catholique, 32, 21-27.

https://doi.org/10.7202/1007327ar

Tous droits réservés ( $@$ Les Éditions Historia Ecclesiæ Catholicæ Canadensis Inc., 1966
Ce document est protégé par la loi sur le droit d'auteur. L’utilisation des services d'Érudit (y compris la reproduction) est assujettie à sa politique d'utilisation que vous pouvez consulter en ligne.

https://apropos.erudit.org/fr/usagers/politique-dutilisation/ 


\section{Les Sœurs du Bon-Pasteur au Saguenay}

Le 3 septembre 1964, notre région célébrait avec un enthousiasme débordant l'arrivée des premières religieuses en terre saguenéenne : cinq sœurs du Bon-Pasteur de Québec, institut fondé par madame François-Xavier Roy, en 1850.

Pour ce centenaire, les plumes les plus solides, les plus élégantes du Saguenay relatèrent, avec force détails, l'événement du 3 septembre 1864. Je cite : les journaux; la revue Saguenéensia; l'album-souvenir Un Siècle d'éducation, rédigé par une équipe de sœurs du Bon-Pasteur, en collaboration avec quelques anciennes élèves.

Comment, après tous ces témoignages, espérer bâtir une œuvre originale ? Aussi, serais-je tentée de reprendre à mon crédit la plainte d'un auteur : "Nous venons trop tard dans un monde trop vieux; tout a été dit. \$

Malgré tout, j'ai décidé de glaner dans le champ de nos annales, d'abord; ensuite, auprès de personnes averties. Mgr Victor Tremblay, président de la Société historique, s'est montré d'une extrême complaisance à mon endroit. Révérende sœur Sainte-Catherine-de-Gênes, assistante et secrétaire provinciale, me fut aussi très secourable. Je tiens à les remercier ici. La revue Saguenéensia, l'Apôtre du Saguenay, me fournirent aussi de beaux, d'abondants épis. Avant de lier ma gerbe, je dus opérer un triage et ne retenir que les plus beaux épis : quelques faits saillants, quelques portraits; je vous les présente.

1862. - Un humble village se blottissait au creux des Laurentides. Son unique rue déroulait son ruban sur les rives majestueuses et sauvages du Saguenay et aboutissait à un modeste temple de bois. Un apôtre à l'âme de feu, "à l'esprit qui calcule et au cœur qui ne calcule pas », était le pasteur aimé de ce bourg perdu, à plus de deux cents milles des grands centres, avec lesquels il ne communiquait que par la rivière. Pendant les rudes mois d'hiver, il était pour ainsi dire coupé du reste du monde. Or, dans ce pays sans chemin de fer, sans routes, sans électricité, sans eau courante, l'apôtre à l'âme de feu (on a reconnu M. le curé Dominique Racine, qui deviendra en 1878 premier évêque de Chicoutimi) rêvait de planter un couvent. Sa vive intelligence, son expérience lui avaient appris « que deux cultures surtout contribuent à former un peuple : : la culture du sol et celle des esprits et des cœurs. La première était en bonne voie : depuis quelque vingt ans, la forêt avait considérablement reculé.

Mais l'éducation de la jeunesse, "malgré des efforts très louables, en était restée au stade élémentaire ». Monsieur le curé Racine rêvait donc d'un couvent, si nécessaire dans un pays jeune, pour former les 
institutrices et les femmes de demain. Il n'était pas le seul à le désirer, puisque le conseil municipal consentit à faire le sacrifice d'une construction, destinée à devenir un hôtel de ville, afin d'y installer religieuses et élèves.

Installation bien rudimentaire, comme en fait foi un article de Mgr Victor Tremblay, dans Saguenéensia (numéro de mars-avril 1960), et je cite : « Ce n'était pas l'étable de Bethléem, mais au témoignage d'une élève des premiers temps, « il fallait voir comme il n'y avait rien ici quand les Sœurs se sont instaīlees. ĪĪere Saint-Ëdouard a tait de ses mains toutes espèces de choses : jusqu'à bûcher, piocher, pelleter, transporter des roches de ses mains, charroyer de la terre dans son tablier, enfin tout, avec les petites filles qui l'aidaient. * Et un ancien, qui avait commencé là ses études, parlait de la petite salle de classe, aménagée sous les toits et où on accédait par un escalier extérieur " tellement dangereux qu'il n'y avait jamais eu d'accident ".

Retournons au 3 septembre 1864. Le rêve devient réalité. Par une maussade journée d'été finissant, sœur Saint-Edouard, supérieure, sœur Saint-Philippe-de-Néri, sœur Sainte-Anne, mettaient le pied sur le sol saguenéen. Elles seront bientôt rejointes par sœur Marie-Joseph et sœur Sainte-Gertrude. Quatre-vingt-six élèves seront confiées à leurs soins.

J'ai eu la bonne fortune de découvrir le nom de deux élèves fondatrices. La première, Suzanne Laforest, seize ans, arrivée de Baie-SaintPaul pendant l'été 1864, soupire après l'ouverture du couvent où elle désire obtenir son parchemin. Ces précisions ont été recueillies de la bouche de Mme Philippe Bergeron, 94 ans, de Jonquière, fille de la précédente.

La seconde, Georgianna Duberger, fait son entrée à notre institut en 1865 et y' prononce ses vœux en 1867, sous le nom de sœur SaintDominique. Le Vieux Couvent (on prit vite l'habitude de désigner ainsi notre première maison à Chicoutimi) bénéficiera de ses services en 1879, comme en font foi les cahiers de Victoria Brassard, mère de Mgr Kérouack, laquelle y mentionne à plusieurs reprises sa chère « tante " Saint-Dominique. On donnait alors à nos religieuses l'appellation de e tante $»$.

J'ai feuilleté moi-même les cahiers de Victoria Brassard. Ils prouvent la qualité de l'enseignement donné au Vieux Couvent en ces temps reculés (1879-1880). La tenue de ces cahiers est impeccable et le contenu intéressant. J'énumère : toisé, algèbre, premier et second degrés; devoirs de littérature : tous les genres sont abordés avec bonheur, même la poésie; des carnets de notes portent : sur la science du ménage, la botanique, l'histoire de l'Eglise, et même un fort cahier de notes de philosophie. Un magnifique herbier, contenant 113 familles de plantes, couronne le tout. Chaque spécimen y est' soigneusement identifié. Il faut dire que le célèbre naturaliste, M. le chanoine Victor Huard, fut chapelain du Vieux Couvent pendant un quart de siècle. 
Ces précisions démontrent que les graduées et les diplômées d'alors possédaient un sérieux bagage de connaissances, soit " des clartés de tout ", comme on disait au Grand Siècle. Aussi leurs " tantes " n'étaient pas les premières venues. Je n'en signalerai que deux.

Sour Saint-Raphaël (Camille Fluet), née à Saint-Jean-Baptiste de Québec, fut élève de notre première école, rue Saint-Amable. Elle y puisa sans doute sa vocation. Supérieure du Vieux Couvent de 1878 à 1884, elle devint secrétaire, puis assistante générale en 1913. Son dévouement, son affabilité l'avaient fait surnommer \& la Charité vivante ». Joignez à cela une remarquable intelligence, une plume alerte (sa correspondance avec Mlle Emilie Tremblay, de Chicoutimi, de 1884 à 1929, en fait foi), et vous comprendrez l'exclamation d'une ancienne élève : "En existe-t-il encore des Mère Saint-Raphaël ? "

Mère Marie-du-Carmel (Emilie Langlois), sa co-paroissienne, née d'une famille en vue, reçut une éducation et une instruction supérieures, qui mirent en valeur des dons de cour et d'esprit exceptionnels. Les dix ans qu'elle passa au Vieux Couvent (1872-1875, 1877-1884), marquèrent un essor dans le niveau intellectuel de l'institution. Elle y amorça même une école ménagère. En effet, Mgr Racine «lui avait mis la navette aux mains, pour les besoins ruraux de cette région». Mais la Providence l'appelait à un rôle moins effacé. Après avoir occupé les postes de supérieure locale et de maîtresse des novices, elle dirigea pendant dix-huit ans les destinées de notre institut, à titre de supérieure générale. Son sage gouvernement affermit notre communauté encore jeune, si bien qu'on l'a surnommée "la seconde fondatrice ".

Mais revenons au Vieux Couvent. L'impulsion donnée par des éducatrices de la trempe des sours Saint-Raphaël et Marie-du-Carmel, lui valut une renommée enviable. Bientôt, les murs devinrent trop étroits, malgré l'addition d'un étage et d'une aile en 1888. On songea à construire une nouvelle institution. Le 12 septembre 1904, l'achat du terrain était conclu. Le Progrès écrivait quelques jours plus tard : - La construction d'un nouveau couvent à Chicoutimi devra régler une question très importante pour notre région : l'établissement d'une Ecole normale. »

Une Ecole normale, le Vieux Couvent en avait jusqu'ici tenu lieu, puisque chaque année un contingent de plus en plus nombreux de ses élèves décrochait le brevet modèle ou élémentaire, au « Bureau central », comme on disait alors. Notre premier couvent possédait donc sa section de pédagogie. La section arts familiaux y était aussi à l'honneur, depuis que Mère Marie-du-Carmel y avait introduit les cours de pratique ménagère. Les arts décoratifs : dessin, peinture, n'étaient pas négligés non plus, comme le prouvent certains travaux très réussis de nos premières élèves. Toutes ces disciplines ne nuisaient en rien au programme régulier des études. Le Vieux Couvent, un siècle avant le rapport Parent, faisait donc figure d'école secondaire polyvalente. C'est le lieu de répéter : « Rien de nouveau sous le soleil.»

Mais, pour faire face au développement rapide de la région, une Ecole normale s'imposait. Mgr Labrecque, évêque de Chicoutimi depuis 
1892, l'avait compris. Le 19 novembre 1906, partant pour Québec, Son Excellence disait aux religieuses du Vieux Couvent : « Il faut que je revienne à Chicoutimi avec mon Ecole normale dans ma poche. Le 27 novembre, Monseigneur avait son Ecole normale. Les travaux allèrent bon train. Le 12 septembre 1907, notre Ecole normale ouvrait ses portes à vingt-huit élèves.

Sa haute direction était confiée à M. l'abbé J.-E. Duchesne. On ne présente pas M. l'abbé Duchesne, ou mieux Mgr Duchesne, aux Chicoutimiens. Pour les personnes étrangères à la région, je signalerai pourtant les hautes qualités de cœur et d'esprit du jeune principal (28 ans). De plus, sa largeur de vue, son dynamisme, son exquise urbanité contribuèrent au bon renom de notre Ecole normale, pendant le quart de siècle où il dirigea les destinées de cette maison, de 1907 à 1914, de 1940 à 1956.

Il convient de dire ici un mot de son méritant successeur (de 1914, à 1940), M. l'abbé Narcisse Desgagné. Ce prêtre modeste sut continuer en profondeur le sillon commencé par son brillant prédécesseur, M. Duchesne. Sa formation austère et virile était acceptée et aimée. Ses normaliennes sentaient, sous la main ferme du maître, battre le cœur du père.

Ces deux ecclésiastiques si dissemblables, l'un s'apparentant assez bien à l'époque romantique, l'autre à l'époque classique, ont' valu à notre Ecole normale un équilibre remarquable dans le domaine de l'éducation et du savoir.

Leurs continuateurs, Mgr Oscar McNicoll et Mgr Floribert Coulombe, le principal actuel, étaient dignes de relever le flambeau.

A côté des principaux, je voudrais esquisser le portrait de deux religieuses qui ont également servi d'assises à notre Ecole normale. La première directrice, Mère Sainte-Cécile (Papillon), légère et menue comme son nom, était douée d'un esprit pétillant et taquin, d'une plume originale et fine. Elle devait occuper, dans notre institut, les plus hautes charges, puisqu'elle devint conseillère générale et maîtresse générale des études.

Sœur Saint-Jean-Berchmans, la supérieure-fondatrice, fut à deux reprises supérieure à l'Ecole normale : de 1907 à 1913, de 1919 à 1925. Quand on l'a connue, on ne peut oublier sa constante sérénité. Aussi son gouvernement était-il marqué par une fermeté douce qui dilatait les âmes. De plus, sœur Saint-Jean-Berchmans était une artiste de talent. Les rares loisirs que lui laissait sa charge, elle les passait à son chevalet. Ces nombreux dons de la nature n'entamaient en rien sa modestie. A son jubilé d'or religieux, elle glissait à l'oreille de sa nièce, sœur SaintAubert, en entrant dans la salle des fêtes : "Tout cela pour la petite fille du quatrième rang de Saint-Nicolas. " Enfin, sœur Saint-JeanBerchmans conserva jusqu'à sa mort (88 ans) une extraordinaire jeunesse d'âme, qui faisait écrire à Mgr Lamarche, quatrième évêque de Chicoutimi : "Une semblable fraîcheur d'âme à un âge aussi avancé est un véritable charisme. "Nous pourrions peindre ici d'autres belles 
figures de vraies religieuses et d'éminentes éducatrices qui ont milité et militent encore à notre Ecole normale. Mais bornons-nous aux pierres d'assises.

Elles étaient de taille; aussi, les lauriers ne se firent-ils pas attendre. Dès 1908, le cours académique, le brevet " $A$ » d'alors, était instauré. Quatre élèves enlevèrent brillamment' le parchemin. Le premier diplôme académique décerné à notre Ecole normale est détenu par révérende sœur Catherine-de-Gênes (Hélène Lajoie), présentement assistante provinciale à Chicoutimi. Si je ne craignais de blesser son humilité, je ferais ici l'éloge de sa haute intelligence, de sa plume originale et humoristique et de son esprit religieux.

Les succès continuèrent les années suivantes. Mais, pendant que la jeune Ecole normale se taillait ainsi une place enviable dans le monde de l'éducation, que devenait le Vieux Couvent? Il avait conservé les élèves externes, l'Ecole normale, à cette époque, n'admettant que des pensionnaires.

Vint la triste année 1912, avec le sinistre du 24 juin, qui réduisit en cendres le quartier est de la ville. Cathédrale, Séminaire, Vieux Couvent furent rasés. Ironie du sort ! quelques jours plus tôt, à l'occasion de la distribution des prix, nos élèves avaient chanté avec allégresse Les feux de la Saint-Jean. Mais où loger les sinistrés? La filiale ouvrit larges ses portes à l'institution mère. De 1912 à 1927, les élèves des deux maisons fraternisèrent.

L'an 1927 vit ressusciter le premier couvent de ses cendres, sous le nom de Pensionnat du Bon-Pasteur. Le nouvel établissement était promis à de très hautes destinées. Le 4 décembre 1936, il était afflié à l'Université Laval, à titre de collège de filles du premier degré. A partir de 1947, le Collège Bon-Pasteur donna le cours classique complet. De son côté, l'Ecole normale décerne le brevet «A » depuis 1959. Que de chemin parcouru à partir des humbles débuts, en 1864!

Entre temps, en juillet 1933, un événement capital pour le BonPasteur saguenéen, s'était produit. Notre communauté ayant pris beaucoup d'expansion, elle obtint de Rome la création de provinces. Notre Ecole normale se vit promue au rang de maison provinciale. Le premier conseil provincial était ainsi composé : Mère Marie-desSéraphins, supérieure provinciale; sœur Saint-Thomas (Maria Paradis), et sœur Sainte-Marguerite (Kilda Renald), assistantes. Ces deux dernières, des Chicoutimiennes, comptaient parmi les premières et les plus brillantes élèves du Vieux Couvent. Quant à Mère Marie-des-Séraphins, elle était bien connue à l'Ecole normale où, en 1911, elle avait remplacé sœur Sainte-Cécile, à titre de directrice. On l'a peinte comme « une semeuse de tout ce qu'il y a de beau, de noble, de pur, dans une vie *. L'éloge résume on ne peut mieux cette nature d'élite, digne de prendre en mains les destinées de la Province du Sacré-Cœur.

Au moment où s'installait ce nouveau mode de gouvernement, la province comptait quatre maisons : le Pensionnat du Bon-Pasteur, remplaçant le Vieux Couvent, l'Ecole normale, l'école du Sacré-Cour, 
ouverte en 1908 dans le quartier ouest de la ville et le couvent NotreDame-du-Carmel, à Jonquière, depuis 1916. A l'heure actuelle elle compte une vingtaine d'établissements. A Chicoutimi, l'école SaintMichel est venue s'ajouter aux institutions précédentes, en 1947; l'école secondaire Laure-Conan, en 1960; le Collège Bon-Pasteur, avenue Lafontaine, en 1964. A Jonquière, notre communauté dirige le Pensionnat Saint-Dominique; en septembre 1964, les écoles secondaires Maria-Chapdelaine et Saint-Laurent remplaçaient le couvent NotreDame-du-Carmel.

Nous comptons aussi, autour du lac Saint-Jean, une belle couronne de maisons : Mistassini, Péribonka, Sainte-Monique-de-Honfleur, SainteJeanne-d'Arc, Saint-Stanislas, Saint-Ambroise. Ajoutons d'autres établissements dans l'Abitibi : à Chibougamau, l'école secondaire Vinette et les écoles primaires Notre-Dame-du-Rosaire et Bon-Pasteur; à Chapais, l'école secondaire Dominique-Savio et les écoles primaires Notre-Damede-Lourdes et Saint-Martin. Nous pouvons maintenant nous écrier à nouveau : "Que de chemin parcouru depuis 1864!" sur le plan horizontal d'abord, puisque nous avons gagné en nombre et en étendue. Quelques statistiques le prouveront éloquemment.

$\begin{array}{lcccc} & \text { Maisons } & \text { Religieuses } & \text { Classes } & \text { Elèves } \\ 1864-1865 & 1 & 5 & 3 & 86 \\ 1964-1965 & 20 & 261 & 278 & 8640\end{array}$

Voyons maintenant le plan vertical. En 1864, nos sœurs ne trouvèrent ici que d'humbles écoles élémentaires. Aussi, Mgr Victor Tremblay avait-il raison d'écrire dans Saguenéensia en août dernier: a L'établissement de cette maison d'éducation (notre premier couvent à Chicoutimi) avait une portée considérable dans la région: elle signifiait plus que l'arrivée d'une communauté de religieuses enseignantes, ce qui est hautement appréciable; elle apportait un degré supérieur d'enseignement et d'éducation et une maison de formation pour ses futures institutrices. \$

C'était déjà un beau palier de franchi, vers les cimes du savoir. Nous avons continué l'ascension. Ainsi, en 1964-1965, nous possédons cinq écoles secondaires, une école normale qui a décerné 3,385 diplômes, dont 60 brevets " $A$ », un collège classique pour jeunes filles avec, à son actif, 89 bachelières ès arts.

Convient-il de nous glorifier pour cet essor inespéré de notre œuvre d'enseignement en terre saguenéenne? "Si je me glorifie moi-même, ma gloire n'est rien ^, dit Jésus. Laissons donc des voix étrangères apprécier notre action auprès de la jeunesse féminine.

En 1917, lors de sa réception à notre Ecole normale, à l'occasion de ses noces d'argent d'épiscopat, Mgr Labrecque s'exprimait ainsi : * Quand je constate la foi vive, les mœurs pures qui honorent et distinguent nos religieuses populations, mon regard observateur en découvre l'une des causes les plus efficaces dans l'œuvre salutaire que vous poursuivez... ' 
Pour sa part, M. l'abbé André Laliberté écrivait dans le Progrès, dont il était rédacteur en 1934 , au $70^{\circ}$ anniversaire de notre arrivée à Chicoutimi : «Elles (les sœurs du Bon-Pasteur) ont formé les jeunes filles, les mères, les institutrices de notre petit pays. On voit leur œuvre dans cette distinction, dans cette religion sincère, dans cet empressement à seconder la charité, qui forment comme les traits caractéristiques des femmes de chez nous.» Il aurait pu ajouter : «Elles ont formé de nombreuses religieuses », puisque dans notre seul institut, 323 religieuses sont sorties de nos écoles, sans compter les nombreuses anciennes qui se sont dirigées vers d'autres congrégations. Mais où les religieux, les religieuses puisent-ils leur vocation, sinon dans le cœur d'une maman chrétienne? Je veux rappeler ici le souvenir d'une mère incomparable, formée à notre Vieux Couvent. Mlle Ariane Ouellet, fille de M. le professeur Elzéar Ouellet, d'Hébertville, par son mariage avec M. Onésime Tremblay, de Saint-Jérôme, a donné à l'Eglise cinq de ses sept enfants : feu M. le chanoine Charles-Elzéar Tremblay, Mgr Victor Tremblay, le R.P. Alphonse Tremblay, c.ss.r., depuis trente-cinq ans missionnaire au Vietnam, le R.P. Laurent Tremblay, l'écrivain bien connu. Son unique fille prenait le voile à l'Hôtel-Dieu Saint-Vallier, sous le nom de sœur Saint-Charles. Honneur à cette chrétienne hors pair, doublée d'une artiste! Il me fut donné d'admirer, au siège de la Société historique, deux de ses peintures au crayon d'un fini exceptionnel.

Une dernière appréciation, parmi d'autres : elle est de Mgr Kéroack, curé de la cathédrale. Lors de la distribution des prix au Pensionnat du Bon-Pasteur, en 1942, il prononçait les paroles suivantes : " Il s'est accompli ici de grandes ouvres... Vous, aujourd'hui, vous rappelez ce qui s'est fait de plus noble et de plus grand : la richesse spirituelle, les biens de l'esprit... Merci (aux Seurs du Bon-Pasteur) de nous avoir donné les études classiques de filles...»

Ajoutons, pour conclure : "Merci à Dieu, surtout, dont nous sommes les servantes... inutiles !»

Oui, lorsque nous contemplons les beaux épis mûris dans notre champ de labeur, nous ne pouvons que nous exclamer : « Le doigt de Dieu est là ! C'est lui qui a tout fait !»

Sœur Marie-dE-SAINTE-IMELda-DU-SaINT-SACREMENT, s.c.i.m. (Imelda Côté)

directrice adjointe à l'Ecole normale du Bon-Pasteur, à Chicoutimi, P.Q. 